\title{
Errata for Bootstrap Methods and their Application
}

\author{
A. C. Davison and D. V. Hinkley
}

May 8, 2006

\section{Subsequent reprints}

The errors below are present in all copies issued up to April 2006.

- Page 268, equation (6.19): the expression should read

$$
p=\frac{\#\left\{z^{*} \geq z_{0}\right\}+1}{R+1}
$$

The errors below are present in all copies issued up to April 2003.

- Page 164, Table 4.3: symbol over fourth column should be $\widehat{\sigma}_{i 0}^{2}$ (missing hat).

- Page 296, line 8: should read ... defined in (6.42), which is minus the bias of ... (insert 'minus' after 'which').

- Page 312, last displayed equation: this should read

$$
r_{j}=\frac{e_{j}}{\left(1-d h_{j}\right)^{1 / 2}}, \quad d=\frac{2 \sum\left(e_{j} / s\right) \psi\left(e_{j} / s\right)}{\sum \dot{\psi}\left(e_{j} / s\right)}-\frac{n \sum \psi^{2}\left(e_{j} / s\right)}{\left\{\sum \dot{\psi}\left(e_{j} / s\right)\right\}^{2}} .
$$

(Factor $n$ has been added in second term of definition of $d$.)

- Page 409, first displayed equation should be:

$$
\tilde{e}_{k}^{*}=2^{-1 / 2}\left(\tilde{X}_{k}+\tilde{X}_{n-k}^{c}\right), \quad k=0, \ldots, n-1,
$$

(replace $\varepsilon$ with $e$ ).

The errors below are present in all copies issued up to February 2002.

- Page 529, second block of code: the function city.sim should be defined as

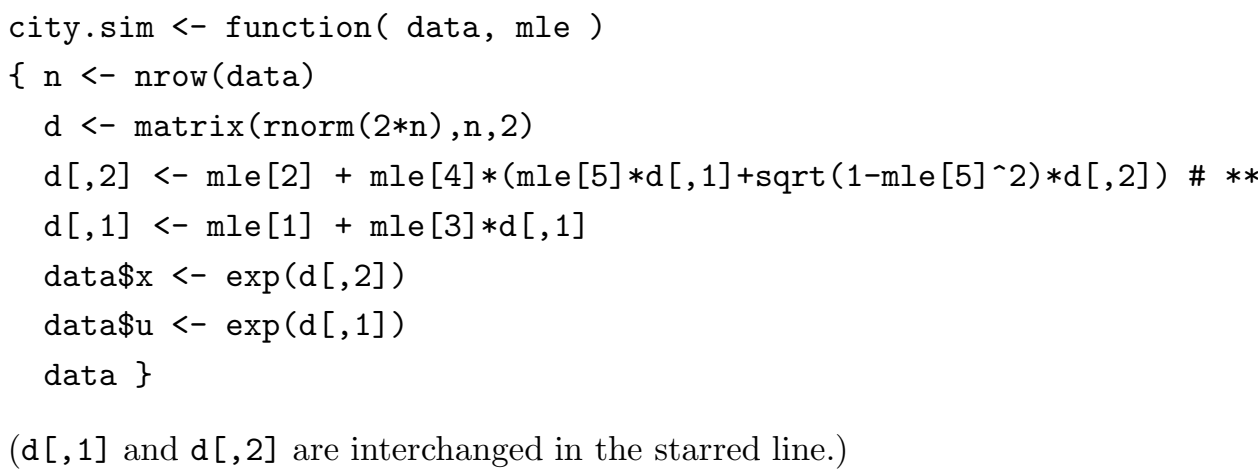

The errors below are present in all copies issued up to December 2000.

- Page 90, top line: should end with $\widehat{y}_{j}^{0}+e_{1}, \ldots, \widehat{y}_{j}^{0}+e_{n-m}$. 


\section{First reprint (1998)}

The errors below are present in the original printing and first reprint.

- Page 28, last line: should read

$$
\operatorname{Pr}(a \leq T-\theta \leq b)=1-2 \alpha \quad \Rightarrow \quad \operatorname{Pr}(T-b \leq \theta \leq T-a) .
$$

- Page 64, third display should be:

$$
L_{t}(y ; F)= \begin{cases}\left\{q_{\alpha}(F)-\mu\right\}(1-2 \alpha)^{-1}, & y<q_{\alpha}(F), \\ (y-\mu)(1-2 \alpha)^{-1}, & q_{\alpha}(F) \leq y<q_{1-\alpha}(F), \\ \left\{q_{1-\alpha}(F)-\mu\right\}(1-2 \alpha)^{-1}, & q_{1-\alpha}(F)<y .\end{cases}
$$

- Page 83, (3.12) should be

$$
\widehat{A}^{0}(y)=\sum_{j: y_{j} \leq y} \frac{d_{j}}{\sum_{k=1}^{n} H\left(y_{k}-y_{j}\right)} .
$$

- Page 86, (3.14) should be

$$
\widehat{A}^{0 *}(y)=\sum_{j=1}^{n} \frac{N_{j}^{*}}{\sum_{k=1}^{n} H\left(y_{k}-y_{j}\right)} .
$$

- Page 86, the second displayed equation should be

$$
d A^{0 *}\left(y_{j}\right)=\frac{N_{j}^{*}}{\sum_{k=1}^{n} H\left(y_{k}-y_{j}\right)}
$$

- Page 188, Practical 3, line 7 of code should be

env $<-$ envelope (exp.boot)

- Page 258, from line before (6.7) should read:

... properties also. Under (6.1),

$$
e_{j}=\varepsilon_{j}-\sum_{k=1}^{n} h_{j k} \varepsilon_{k}
$$

where

$$
h_{j k}=n^{-1}+\frac{\left(x_{j}-\bar{x}\right)\left(x_{k}-\bar{x}\right)}{S S_{x}} .
$$

The quantities $h_{j j}$ are known as leverages, ...

- Page 342, first displayed equation should be

$$
y_{+, j}^{*}=\sum_{k \text { unobs }} y_{j k}^{*}, \quad \widehat{\mu}_{+, j}^{*}=\exp \left(\widehat{\alpha}_{j}^{*}\right) \sum_{k \text { unobs }} \exp \left(\widehat{\beta}_{k}^{*}\right), \quad j=1, \ldots, 38,
$$

(hats added to $\alpha_{j}^{*}$ and $\beta_{k}^{*}$ ).

- Page 350, the denominators in (7.17), (7.18) and the following displayed equation should be

$$
\sum_{k=1}^{n} H\left(y_{k}-y_{j}\right) \exp \left(x_{k}^{\mathrm{T}} \beta\right), \quad \sum_{k=1}^{n} H\left(y_{k}-y_{j}\right) \exp \left(x_{k}^{\mathrm{T}} \widehat{\beta}\right), \quad \sum_{k=1}^{n} H\left(y_{k}-y_{j}\right) \exp \left(x_{k}^{\mathrm{T}} \widehat{\beta}\right)
$$

(replacement of $y_{j}-y_{k}$ with $y_{k}-y_{j}$ ). 
- Page 501, first line after second displayed equation: should be 'has an approximate chi-squared distribution with one degree of freedom.'

- Page 522, line 2 of text: Change langauge to language.

- Page 550, line -13: Change Example 10.4 to Problem 10.4.

\section{Original printing}

The errors below were corrected in the first (1998) reprint.

- Page 31 , line $-7: \ldots$ assume that the estimate $T \ldots$

- Page 32, first two paragraphs under heading 'Parametric problems' should be:

In parametric problems $\widehat{F} \equiv F_{\widehat{\psi}}$ and $F \equiv F_{\psi}$ have the same form, differing only in parameter values. The notion of a pivot is quite simple here, meaning constant behaviour under all values of the model parameters. More formally, we define a pivot as a function $Q=q(T, \theta)$ whose distribution does not depend on the value of $\psi$ : for all $q$,

$$
\operatorname{Pr}\{q(T, \theta) \leq q \mid \psi\}
$$

is independent of $\psi$.

One can check, sometimes theoretically and always empirically, whether or not a particular quantity $Q$ is exactly or nearly pivotal, by examining its behaviour under the model form with varying parameter values. For example, in the context of Example 1.1, we could simultaneously examine properties of $T-\theta, \log T-\log \theta$ and the studentized version of the former, by simulation under several exponential models close to the fitted model. This might result in plots of variance or selected quantiles versus parameter values, from which we could diagnose the non-pivotal behaviour of $T-\theta$ and the pivotal behaviour of $\log T-\log \theta$.

- Page 155, equation (4.19): $\leq$ should be $\geq$

- Page 187, line -4: Change 'zero correlation between' to 'independence of'.

- Page 188: the code for Practical 3 can be amended to:

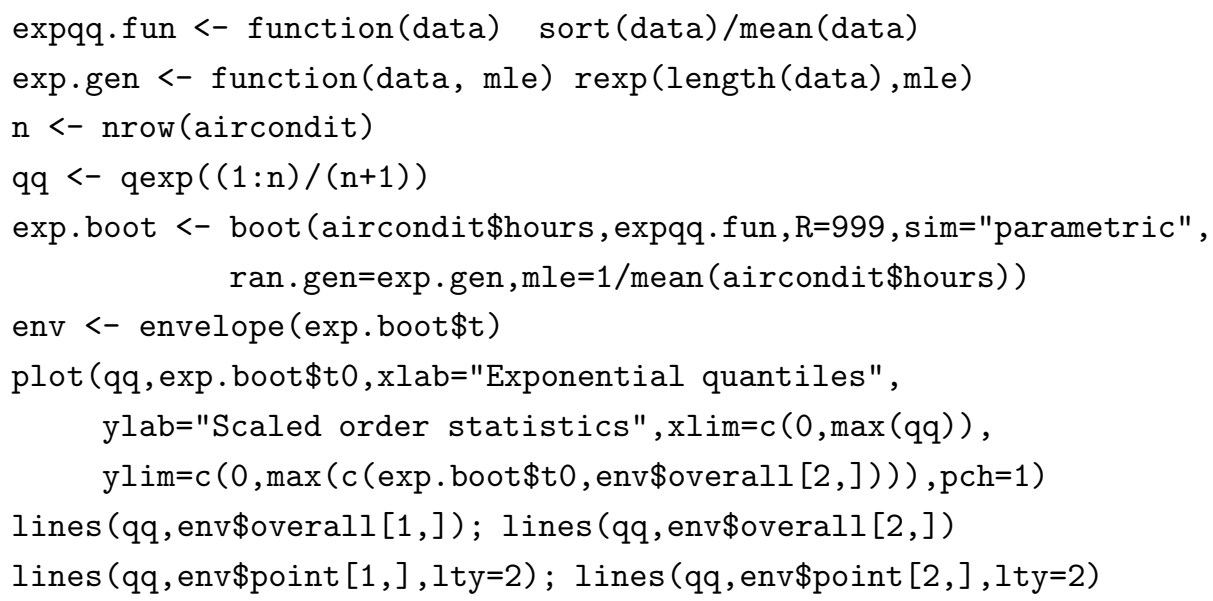


(Minor changes to expqq.fun and exp.boot, which should not change any results.)

- Page 298: right-hand side of third displayed equation should be

$$
\sigma^{2}\left\{1+(3 w-1) q n^{-1}\right\}+O\left(n^{-2}\right),
$$

- Page 303, equation (6.58) should be

$$
n \widehat{\Delta}_{C V}(M) \doteq \varepsilon^{T}\left(I-H_{M}\right) \varepsilon+2 q_{M} \sigma^{2}+\mu^{T}\left(I-H_{M}\right) \mu .
$$

- Page 350: the subscripts on $\beta$ and $\widehat{\beta}$ in equations (7.17), (7.18), and the display just after (7.18) should be on the $x$, respectively giving

$$
\frac{\exp \left(x_{j}^{T} \beta\right)}{\sum_{k=1}^{n} H\left(y_{j}-y_{k}\right) \exp \left(x_{k}^{T} \beta\right)},
$$

and

$$
\widehat{A}^{0}(y)=\sum_{j: y_{j} \leq y} \frac{d_{j}}{\sum_{k=1}^{n} H\left(y_{j}-y_{k}\right) \exp \left(x_{k}^{T} \widehat{\beta}\right)},
$$

and

$$
d \widehat{A}^{0}\left(y_{j}\right)=\frac{d_{j}}{\sum_{k=1}^{n} H\left(y_{j}-y_{k}\right) \exp \left(x_{k}^{T} \widehat{\beta}\right)} .
$$

- Page 420, line just above fourth display should read and $(R+1)(1-\alpha)$ th ordered values of (insertion of 'and $(R+1)(1-\alpha)$ th' at beginning of line).

- Page 480, equation (9.47) should be

$$
\frac{d^{2} K(\xi ; t, s)}{d \xi d s^{T}}=n \sum_{j=1}^{n} p_{j}^{\prime}(t, s)\left\{\frac{d a_{j}(t, s)}{d s^{T}}+a_{j}(t, s) \xi^{T} \frac{d a_{j}(t, s)}{d s^{T}}\right\}
$$

(addition of $(t, s)$ just before $\xi^{T}$ on right).

- Page 481, last display should be

(the second derivatives with respect to $z$ are not needed).

$$
\frac{d a_{j}(z, v)}{d z}, \quad \frac{d a_{j}(z, v)}{d v}, \quad \frac{d^{2} a_{j}(z, v)}{d v^{2}},
$$

- Page 526, line -9: should be

$\{\mathrm{d}<-\operatorname{data}[i]$,

(final ] ).

- Page 527, line 5: code segment should be

boot array (city $\cdot$ boot, indices=T)

(not index=T).

- Page 534, line -9: Change 'zero correlation between' to 'independence of'.

- Page 535, line 2: Change 'zero correlation' to 'independence'.

- Page 559: The order of the references Efron (1981a) and Efron (1981b) should be reversed, so Efron (1981a) is the JASA censored data paper, and Efron (1981b) is the CJS paper on standard errors and confidence. 